\title{
Prevalencia de consumo riesgoso y dañino de alcohol en derechohabientes del Instituto Mexicano del Seguro Social
}

\author{
Juana Inés de la Cruz Morales-García, MC, MSP, ${ }^{(1)}$ Irma H Fernández-Gárate, MCP, MSP, \\ Hugo Tudón-Garcés, FM, ${ }^{(1)}$ Jorge Escobedo-de la Peña, MC, MSP, M en $C, F A C P_{1}^{(1)}$ \\ Angel Zárate-A guilar, MC, ${ }^{(2)}$ Mario Madrazo-N avarro, MC.(3)
}

Morales-García JIC, Fernández-Gárate IH,
Tudón-Garcés H, Escobedo-de la Peña J,
Zárate-Aguilar A, Madrazo-Navarro M.
Prevalencia de consumo riesgoso
y dañino de alcohol en derechohabientes
del Instituto Mexicano del Seguro Social.
Salud Publica Mex 2002;44:113-121.

Salud Publica Mex 2002;44:113-121.

El texto completo en inglés de este articulo está

\section{Resumen}

Objetivo. Medir la prevalencia de consumo riesgoso y dañino de alcohol en la población amparada por el Instituto Mexicano del Seguro Social (IMSS). Material y métodos. En un estudio transversal se entrevistaron 45117 derechohabientes del IMSS, en las 36 delegaciones del país. Se aplicó un cuestionario estructurado y autoaplicable a cada sujeto usando el instrumento de tamizaje AUDIT (Alcohol Use Disorder Identification Test), el cual consta de 10 preguntas. Las tres primeras se relacionan con la cantidad y frecuencia del consumo de alcohol, las tres siguientes evalúan la dependencia y las cuatro últimas se refieren a problemas causados por el alcohol. Cada pregunta consta de tres a cinco opciones, a las que se les asignan valores progresivos de cero a cuatro. Se estimó la prevalencia de consumo, con intervalos de confianza al 95\% (IC 95\%). Resultados La prevalencia de consumo problema de alcohol (riesgoso y dañino) fue de $12.8 \%$ (IC 95\% 12.5-13.2). El consumo fue mayor en hombres (22.2\%; IC 95\% 21.7-22.8) que en mujeres (3.4\%; IC 95\% 3.1-3.6). En los hombres se observó un efecto de edad, mientras que en las mujeres el consumo por edad fue más homogéneo. En ambos grupos el consumo fue más importante en la edad productiva. Conclusiones La prevalencia de consumo de alcohol que constituye un problema para la salud, es elevada en la población dere-
Morales-García JIC, Fernández-Gárate IH, Tudón-Garcés H, Escobedo-de la Peña J, Zárate-Aguilar A, Madrazo-Navarro M. The prevalence of hazardous and harmful use of alcohol among the insured population of Instituto Mexicano del Seguro Social. Salud Publica Mex 2002;44:113-121.

The English version of this paper is available at: http://www.insp.mx/salud/index.html

\begin{abstract}
A bstract
Objective. To estimate the prevalence of hazardous and har mful alcohol consumption among the insured population of Instituto Mexicano del Seguro Social (Mexican Social Security Institute, IMSS). Material and Methods. A crosssectional survey was conducted in 45,117 insured subjects from Mexico's 36 political districts; study subjects were interviewed using a structured and self-applied questionnaire on alcohol consumption, using the ten-item screening instrument AUDIT (Alcohol Use Disorder Identification Test). The first three questions refer to the amount and frequency of drinking, the following three question to alcohol dependence, and the last four to alcohol-related problems. Each item has three to five options and each is scored progressively from zero to four. The prevalence of alcohol consumption was estimated with $95 \%$ confidence intervals. Results. The prevalence of problem drinkers (hazardous and harmful) was 12.8\% (IC 95\% 12.5-13.2); it was higher in men (22.2\%; IC 95\% 21.7-22.8) than in women (3.4\%; IC 95\% 3.1-3.6). An age effect was observed in men, while in women alcohol consumption was more homogeneous. In both genders consumption was higher in productive-age groups. Conclusions. The prevalence of hazardous and harmful alcohol consumption is high in the insured population of the IMSS in Mexico.There is a need
\end{abstract}

(1) División de Epidemiología, Coordinación de Salud Comunitaria, Instituto Mexicano del Seguro Social, (IMSS), México, D.F., México.

(2) Coordinación de Salud Comunitaria, IMSS, México, D.F., México.

(3) Dirección de Prestaciones Médicas, IMSS, México, D.F., México.

Fecha de recibido: 29 de enero de 2001 - Fecha de aprobado: 18 de octubre de 2001

Solicitud de sobretiros: D ra. Juana Inés de la Cruz Morales García. Av. Insurgentes Sur 253-40. piso, colonia Roma, 06700 México, D.F., México. Correo electrónico: jorgeep@ servidor.unam.mx 
chohabiente del IMSS. Es necesario desarrollar políticas y programas de salud específicos, para disminuir este grave problema. El texto completo en inglés de este artículo está disponible en: http://www.insp.mx/salud/index.html

Palabras clave: alcoholismo; AUDIT; consumo riesgoso de alcohol; consumo dañino de alcohol; México to implement health policies and health programs to diminish this serious health problem. The English version of this paper is available at: http://www.insp.mx/salud/index.html

Key words: alcoholism;AUD IT; hazardo us alcohol consumption; harmful alcohol consumption; Mexico
$\mathrm{E}$ 1 consumo de alcohol es un problema de salud ge neralizado y común en toda sociedad. Está asociado con violencia familiar, riñas en las calles, accidentes y muertes violentas. A su vez el alcohol entra a todos los tejidos del organismo y afecta la mayoría de las funciones vitales. Desplaza a los nutrientes normales y produce desnutrición. Sus efectos tóxicos dañan al hígado, producen gastritis erosiva aguda, pancreatitis y, a largo plazo miocardiopatía. El consumo de $20 \mathrm{~g}$ de alcohol absoluto en mujeres y de 40 en hombres, es un factor de riesgo para accidentes, lesiones y alteraciones crónicas de salud; sin embargo, los problemas atribuidos al alcoholismo son subestimados. ${ }^{1,2}$ El consumo de alcohol se ha incrementado más en familiares de alcohólicos que entre parientes de no alcohólicos, sugiriendo un factor genético en su etiología. En la encuesta nacional de salud de los Estados Unidos de América (EUA), realizada en 1988, 36\% de los hombres y 39\% de las mujeres informaron antecedentes familiares de alcoholismo. ${ }^{3}$ En dicha encuesta la prevalencia nacional encontrada para consumo de alcohol fue de $51.6 \%$; los menores de 44 años consumieron más alcohol y el porcentaje de consumo fue mayor en los hombres $(63.6 \%)$ y en la raza blanca $(54 \%){ }^{4}$

En pacientes hospitalizados la prevalencia de alcoholismo es alta y oscila de 15 al 30\%. En los servicios de urgencias 18\% de los mayores de 16 años son bebedores, sobre todo los hombres. ${ }^{6}$

En México se han hecho tres Encuestas Nacionales de Adicciones (ENA). En 1998 la prevalencia de consumo nacional de alcohol fue de $58.1 \%$ (77.0\% en hombres y $44.6 \%$ en mujeres). ${ }^{7}$ Esta prevalencia fue menor que la observada cinco años antes. En 1993 se informó una prevalencia global de $66.5 \%$. Mientras que la prevalencia en hombres no mostró gran cambio para 1998 (se había notificado una prevalencia de $77.2 \%$ en 1993), la correspondiente en mujeres fue sensiblemente menor a $57.5 \%$ estimado en $1993 .{ }^{8}$ Ambas estimaciones, sin embargo, son mayores que las observadas en la primera ENA realizada en 1990, en la cual $73.4 \%$ de los hombres y $36.5 \%$ de las mujeres consumían bebidas alcohólicas. ${ }^{9}$
Existen diversos instrumentos de estudio para identificar el consumo del alcohol. La mayoría son muy sensibles para detectar problemas avanzados de alcoholismo, pero son menos confiables para identificar etapas incipientes del consumo de alcohol. En 1982 la Organización Mundial de la Salud (OMS) diseñó un instrumento de tamizaje simple llamado AUDIT (Alcohol Use Disorder Identification Test) para identificar personas cuyo consumo de alcohol se haya convertido en un problema de salud, ya sea porque el consumo entrañe riesgos o daños a la salud, o bien dependencia. El AUDIT consta de 10 preguntas seleccionadas con base en su reproducibilidad y correlación con el consumo de alcohol. El AUDIT es un cuestionario frecuentemente utilizado y aplicado por su capacidad para medir el consumo problema de alcohol, dada su alta sensibilidad (92\%) y especificidad (94\%). En estudios de seguimiento se ha probado su capacidad predictiva de problemas médicos, trastornos sociales e incluso mortalidad, relacionados con el consumo de alcohol. $^{10}$

Las ENA en México no han empleado el AUDIT como instrumento de medición del alcoholismo. En consecuencia, no se conoce nacionalmente la magnitud del consumo riesgoso o dañino de alcohol, el cual está relacionado con problemas en la salud, accidentes y violencias, entre otras condiciones. Con el fin de conocer la prevalencia del consumo problema de alcohol (riesgoso o dañino) en la población amparada por el Instituto Mexicano del Seguro Social (IMSS), la mayor institución de seguridad social en México, que cubre más de la mitad de la población, se llevó a cabo una encuesta nacional en población derechohabiente del Instituto.

\section{Material y métodos}

Se realizó un estudio transversal en la población derechohabiente del IMSS, usuaria de los servicios de atención médica, del 1 de noviembre de 1993 al 31 de agosto de 1994, con el fin de medir la prevalencia de consumo problema de alcohol, a través del instrumento de 
medición AUDIT, diseñado por la OMS. Se incluyeron derechohabientes de las 36 delegaciones. Cada delegación corresponde a un estado de la República, salvo el Distrito Federal, que tiene cuatro delegaciones, y el estado de Veracruz, que está formado por dos.

En el país se distribuyeron 50000 cuestionarios estructurados y autoaplicables, que incluían información sobre edad, sexo y lugar de residencia del derechohabiente entrevistado, así como el cuestionario de AUDIT (ver Anexo $\mathrm{N}^{\circ}$ 1) para evaluar el consumo de alcohol. De acuerdo con el número de derechohabientes en cada delegación, se asignaron proporcionalmente el número correspondiente de cuestionarios, y el epidemiólogo coordinador de los servicios de salud pública, o el responsable designado, los distribuyó también de manera proporcional a cada una de las unidades médicas de los tres niveles de atención de la delegación. En las unidades, el epidemiólogo o el responsable designado, coordinó la distribución diaria de los cuestionarios hasta cumplir con la cuota establecida para dicha unidad, entre los usuarios de los servicios de los dos turnos de la consulta externa y de urgencias, mayores de 11 años de edad, de uno u otro sexo y que aceptaron participar en el estudio. En los menores de edad se solicitó el consentimiento del acompañante mayor de edad, y en el total de los entrevistados se garantizó la confidencialidad de sus respuestas.

Para el análisis se utilizó la información disponible en cada uno de los cuestionarios, toda vez que la participación había sido voluntaria y el cuestionario autoadministrado. En ocasiones el sujeto contestaba la información relevante sobre el consumo de alcohol, pero por ejemplo omitía contestar preguntas sobre edad o sexo. La información presentada en la sección de resultados corresponde a la proporcionada en cada una de las preguntas y el total en cada una de las variables puede ser, en consecuencia, variable.

A los entrevistados se les solicitó información sobre edad y sexo al inicio del cuestionario. Se incluyó la escala de AUDIT que consiste en 10 preguntas que abordan el consumo, frecuencia e intensidad del consumo del alcohol, instrumento desarrollado por la OMS para estudios de tamizaje sobre el consumo riesgoso de alcohol en unidades de primer nivel de atención (Anexo 1). Las tres primeras preguntas están relacionadas con la cantidad y frecuencia del consumo de alcohol, por lo que permiten la estimación de la abstinencia o el consumo riesgoso. Las tres siguientes preguntas están relacionadas con la dependencia al alcohol, mientras que las preguntas 7 y 8 son dirigidas al consumo dañino y las dos últimas se relacionan con problemas causados por alcohol, incluyendo reacciones psicológicas adversas. Cada pregunta tiene de tres a cinco opciones que discriminan en forma categórica la frecuencia y cantidad de consumo. A cada opción se le da un valor numérico partiendo de cero en el nivel inferior y en orden progresivo hasta dos o cuatro puntos (dependiendo del número de opciones). La sumatoria de los puntos aportados con cada pregunta da como resultado el índice de la escala AUDIT, con un máximo posible de 39 puntos. Con el fin de caracterizar el consumo de alcohol que constituye un problema para la salud, la OMS sugirió inicialmente un punto de corte de 11 puntos, ${ }^{11}$ el cual se cambió más tarde a ocho puntos de la escala de AUDIT. Este último punto de corte es más sensible (95\%) que el anterior, aunque menos específico $(88 \%)$, pero está más acorde con la reducción en los límites de consumo seguro de alcohol. ${ }^{12}$ Se considera consumo seguro de alcohol o "normal", cuando el puntaje en el AUDIT es menor de nueve puntos. A su vez se establecieron dos puntos de corte adicionales dentro del consumo "problema" de alcohol, ${ }_{1}^{13}$ considerando una calificación entre 9 y 18 puntos como consumo "riesgoso" y cuando la calificación fue mayor a 18 puntos se consideró como consumo "dañino". De acuerdo con estos criterios, el consumo "riesgoso" está relacionado con un mayor riesgo de daño físico o psicológico secundario al consumo de alcohol, mientras que el concepto de "dañino" se refiere más a la presencia de complicaciones físicas o psicológicas relacionadas con su ingesta.

Se estimó la prevalencia global de consumo "problema" de alcohol, así como la de las diferentes categorías señaladas (riesgoso y dañino). Se estimó la prevalencia por grupo de edad y sexo y la correspondiente a cada una de las delegaciones del Instituto. Se estimaron intervalos de confianza a $95 \%$ para la prevalencia de consumo, bajo el método exacto. Se graficó en un mapa de la República Mexicana la distribución por terciles de consumo problema de alcohol (AUDIT superior a ocho puntos).

\section{Resultados}

Se aplicaron 45117 cuestionarios en las 36 delegaciones del Instituto, de los cuales se eliminaron 4656 $(10.3 \%)$ porque no fueron devueltos o porque la información proporcionada no era adecuada para el análisis. En total se estudiaron 40461 personas, hombres y mujeres en igual proporción.

La prevalencia de consumo de alcohol que constituye un problema para la salud fue de $12.8 \%$ (IC 95\% 12.5-13.2) en el ámbito nacional (cuadro I). El consumo osciló de 7.5\% (IC 95\% 5.3-10.3) en la delegación Chiapas a 28.0\% (IC 95\% 21.0-34.9) en la delegación de 


\section{Cuadro I \\ Consumo problema de alcohol (RIESgoso y dañIno), SEgún delegación. Instituto Mexicano del Seguro Social, 1994}

\begin{tabular}{|c|c|c|c|c|c|c|c|c|c|c|}
\hline \multirow{4}{*}{ Delegación } & \multicolumn{6}{|c|}{ Consumo } & \multirow{3}{*}{\multicolumn{2}{|c|}{ Total }} & \multirow{3}{*}{\multicolumn{2}{|c|}{$\begin{array}{l}\text { Encuestas } \\
\text { Total }\end{array}$}} \\
\hline & \multirow{2}{*}{\multicolumn{2}{|c|}{ Seguro }} & \multirow{2}{*}{\multicolumn{4}{|c|}{ Problema }} & & & & \\
\hline & & & & & & & & & & \\
\hline & № & $\%$ & № & $\%$ & № & $\%$ & № & $\%$ & № & $\%$ \\
\hline Aguascalientes & 438 & 85.7 & 55 & 10.8 & 18 & 3.5 & 73 & 14.3 & 511 & 1.1 \\
\hline Baja California & 1156 & 84.3 & 177 & 12.9 & 39 & 2.8 & 216 & 15.7 & 1375 & 3.0 \\
\hline Baja C alifornia Sur & 116 & 72.0 & 36 & 22.4 & 9 & 5.6 & 45 & 28.0 & 161 & 0.4 \\
\hline Campeche & 210 & 86.1 & 31 & 12.7 & 3 & 1.2 & 34 & 13.9 & 244 & 0.5 \\
\hline Coahuila & 1673 & 85.2 & 227 & 11.6 & 63 & 3.2 & 290 & 14.8 & 1963 & 4.4 \\
\hline Colima & 426 & 91.2 & 32 & 6.9 & 9 & 1.9 & 41 & 8.8 & 467 & 1.0 \\
\hline Chiapas & 432 & 92.5 & 27 & 5.8 & 8 & 1.7 & 35 & 7.5 & 467 & 1.0 \\
\hline Chihuahua & 1281 & 84.9 & 185 & 12.3 & 42 & 2.8 & 227 & 15.1 & 1508 & 3.3 \\
\hline Durango & 1656 & 86.5 & 201 & 10.5 & 58 & 3.0 & 259 & 13.5 & 1915 & 4.2 \\
\hline Guanajuato & 1532 & 86.9 & 185 & 10.5 & 46 & 2.6 & 231 & 13.1 & 1763 & 3.9 \\
\hline Guerrero & 1702 & 90.1 & 151 & 8.0 & 36 & 1.9 & 187 & 9.9 & 1889 & 4.2 \\
\hline Hidalgo & 1959 & 89.8 & 168 & 7.7 & 55 & 2.5 & 223 & 10.2 & 2182 & 4.8 \\
\hline Jalisco & 183 & 83.9 & 23 & 10.6 & 12 & 5.5 & 35 & 16.1 & 218 & 0.5 \\
\hline México & 1200 & 86.8 & 148 & 10.7 & 34 & 2.5 & 182 & 13.2 & 1382 & 3.1 \\
\hline Michoacán & 492 & 87.7 & 49 & 8.7 & 20 & 3.6 & 69 & 12.3 & 561 & 1.2 \\
\hline Morelos & 437 & 84.2 & 67 & 12.9 & 15 & 2.9 & 82 & 15.8 & 519 & 1.2 \\
\hline N ayarit & 3465 & 89.1 & 331 & 8.5 & 95 & 2.4 & 426 & 10.9 & 3891 & 8.6 \\
\hline Nuevo León & 5039 & 88.1 & 559 & 9.8 & 124 & 2.2 & 683 & 11.9 & 5722 & 12.7 \\
\hline 0 axaca & 862 & 87.1 & 101 & 10.2 & 27 & 2.7 & 128 & 12.9 & 990 & 2.2 \\
\hline Puebla & 549 & 88.8 & 61 & 9.9 & 8 & 1.3 & 69 & 11.2 & 618 & 1.4 \\
\hline Q uerétaro & 341 & 76.8 & 74 & 16.7 & 29 & 6.5 & 103 & 23.2 & 444 & 1.0 \\
\hline Q uintana Roo & 3073 & 90.7 & 247 & 7.3 & 68 & 2.0 & 315 & 9.3 & 3388 & 7.5 \\
\hline San Luis Potosí & 286 & 79.4 & 55 & 15.3 & 19 & 5.3 & 74 & 20.6 & 360 & 0.8 \\
\hline Sinaloa & 2440 & 85.2 & 337 & 11.8 & 87 & 3.0 & 424 & 14.8 & 2864 & 6.3 \\
\hline Sonora & 671 & 84.9 & 102 & 12.9 & 17 & 2.2 & 119 & 15.1 & 790 & 1.8 \\
\hline Tabasco & 254 & 76.7 & 62 & 18.7 & 15 & 4.5 & 77 & 23.3 & 331 & 0.7 \\
\hline Tamaulipas & 1074 & 86.4 & 143 & 11.5 & 26 & 2.1 & 169 & 13.6 & 1243 & 2.8 \\
\hline Tlaxcala & 1263 & 87.4 & 140 & 9.7 & 42 & 2.9 & 182 & 12.6 & 1445 & 3.2 \\
\hline Veracruz N orte & 567 & 86.4 & 77 & 11.7 & 12 & 1.8 & 89 & 13.6 & 656 & 1.5 \\
\hline Veracruz Sur & 228 & 87.7 & 25 & 9.6 & 7 & 2.7 & 32 & 12.3 & 260 & 0.6 \\
\hline Yucatán & 1423 & 88.3 & 163 & 10.0 & 26 & 1.6 & 189 & 11.7 & 1612 & 3.6 \\
\hline Zacatecas & 353 & 85.7 & 42 & 10.2 & 17 & 4.1 & 59 & 14.3 & 412 & 0.9 \\
\hline Distrito Federal 1 & 1019 & 86.1 & 135 & 11.4 & 30 & 2.5 & 165 & 13.9 & 1184 & 2.6 \\
\hline Distrito Federal 2 & 538 & 82.8 & 78 & 12.0 & 34 & 5.2 & 112 & 17.2 & 650 & 1.4 \\
\hline Distrito Federal 3 & 653 & 86.0 & 77 & 10.1 & 29 & 3.8 & 106 & 14.0 & 759 & 1.7 \\
\hline Distrito Federal 4 & 329 & 88.2 & 33 & 8.8 & 11 & 2.9 & 44 & 11.8 & 373 & 0.8 \\
\hline Total & 39323 & 87.2 & 4604 & 10.2 & 1190 & 2.6 & 5794 & 12.8 & 45117 & 100.0 \\
\hline
\end{tabular}

Baja California Sur. La prevalencia de consumo riesgoso fue de $10.2 \%$ (IC 95\% 9.9.-10.5), mientras que la correspondiente al consumo dañino fue de $2.6 \%$ (IC $95 \%$ 2.5-2.8). En el cuadro I se observa también la distribución de sujetos encuestados por delegación.

En la figura 1 se observa la distribución geográfica por terciles de consumo problema de alcohol. Aun cuando el patrón es heterogéneo, se observa una tendencia a mayor consumo en los estados del norte del país y menor en los del sur y del oriente.

El consumo de alcohol fue francamente mayor en los hombres (22.2\%; IC 95\% 21.7-22.8), que en las mujeres (3.4\%; IC 95\% 3.1-3.6). Hubo un mayor consumo riesgoso que dañino en uno $u$ otro sexo. Los hombres 


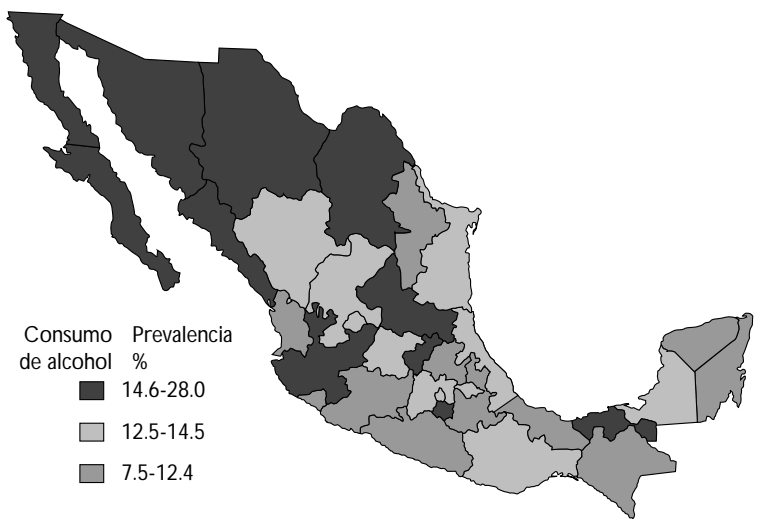

Figura 1. Distribución geográfica de la prevalencia DE CONSUMO PROBLEMA de ALCOHOL, SEgún Delegación. Instituto Mexicano del Seguro Social, 1994

tuvieron un consumo riesgoso mayor (17.7\%; IC 95\% 17.2-18.2)) que las mujeres (2.7\%; IC 95\% 2.5-2.9) y un consumo dañino también mayor (4.5\% vs. $0.7 \%$ ) como puede verse en los cuadros II y III.

En los hombres hubo un claro efecto de edad en la frecuencia de consumo de alcohol considerado como problema para la salud. El consumo se incrementó con la edad, hasta los 50 años, en lo que se observa un descenso. Un patrón similar se observa en el consumo dañino de alcohol. En el consumo riesgoso el patrón es más irregular. La prevalencia es muy similar en los grupos de 20 a 49 años, edad en la cual se empieza a observar un descenso en la frecuencia de consumo riesgoso. Llama la atención que en el grupo de 45 a 49 años uno de cada 15 individuos tiene un consumo dañino de alcohol. De hecho en este grupo de edad, en uno de cada cuatro individuos el consumo de alcohol constituye un problema para la salud (cuadro II). En las mujeres el patrón de consumo es más homogéneo en los diferentes grupos de edad y, como se señaló anteriormente, menor que en el hombre (cuadro III).

Llama la atención que la prevalencia de consumo riesgoso y dañino en el grupo de edad más joven (12 a 19 años), es de 4.4\% (IC 95\% 3.4-5.5) en las mujeres y de $17.9 \%$ (IC $95 \% 15.9-19.9$ ) en los hombres, lo cual indudablemente traduce la importancia de esta adicción en los jóvenes estudiados (cuadros II y III).

\section{Discusión}

La identificación de un consumo problema de alcohol no deja de entrañar dificultades técnicas y éticas. Se ha señalado que un consumo moderado de alcohol puede ser saludable y proteger al individuo contra la cardiopatía isquémica. ${ }^{14}$ De esta forma, una política de salud pudiera estar orientada a promover un consumo moderado de alcohol. La disyuntiva se genera al establecer el punto de corte entre consumo moderado o

Cuadro II

Consumo problema de Alcohol (RIESgoso y dAÑINo), POR GRUPO DE EDAD EN hOMBRes. Instituto Mexicano del Seguro Social, 1994

\begin{tabular}{|c|c|c|c|c|c|c|c|c|c|c|}
\hline \multirow{4}{*}{$\begin{array}{c}\text { Grupo } \\
\text { de edad }\end{array}$} & \multicolumn{6}{|c|}{ Consumo } & & & & \\
\hline & \multirow{2}{*}{\multicolumn{2}{|c|}{ Seguro }} & \multicolumn{4}{|c|}{ Problema } & \multirow{2}{*}{\multicolumn{2}{|c|}{ Total }} & \multirow{2}{*}{\multicolumn{2}{|c|}{$\begin{array}{l}\text { Encuestas } \\
\text { Total }\end{array}$}} \\
\hline & & & & & & & & & & \\
\hline & № & $\%$ & № & $\%$ & № & $\%$ & № & $\%$ & № & $\%$ \\
\hline $12-19$ & 1144 & 82.1 & 214 & 15.4 & 15.4 & 2.6 & 250 & 17.9 & 1394 & 6.89 \\
\hline $20-24$ & 2425 & 77.5 & 582 & 18.6 & 124 & 4.0 & 706 & 22.5 & 3131 & 15.48 \\
\hline $25-29$ & 2467 & 76.1 & 625 & 19.3 & 151 & 4.7 & 776 & 23.9 & 3243 & 16.03 \\
\hline $30-34$ & 2163 & 75.2 & 582 & 20.2 & 133 & 4.6 & 715 & 24.8 & 2878 & 14.23 \\
\hline $35-39$ & 1891 & 75.9 & 475 & 19.1 & 126 & 5.1 & 601 & 24.1 & 2492 & 12.32 \\
\hline $40-44$ & 1455 & 76.5 & 350 & 18.4 & 96 & 5.0 & 446 & 23.5 & 1901 & 9.40 \\
\hline $45-49$ & 1003 & 73.6 & 269 & 19.8 & 90 & 6.6 & 359 & 26.4 & 1362 & 6.73 \\
\hline $50-54$ & 890 & 78.1 & 186 & 16.3 & 64 & 5.6 & 250 & 21.9 & 1140 & 5.64 \\
\hline $55-59$ & 725 & 83.3 & 104 & 12.0 & 41 & 4.7 & 145 & 16.7 & 870 & 4.30 \\
\hline $60-64$ & 649 & 83.1 & 104 & 13.3 & 28 & 3.6 & 132 & 16.9 & 781 & 3.86 \\
\hline $65-69$ & 464 & 87.7 & 52 & 9.8 & 13 & 2.5 & 65 & 12.3 & 529 & 2.62 \\
\hline $70-79$ & 456 & 89.8 & 39 & 7.7 & 13 & 2.6 & 52 & 10.2 & 508 & 2.51 \\
\hline Total & 15732 & 77.8 & 3582 & 17.7 & 915 & 4.5 & 4497 & 22.2 & 20229 & 100.0 \\
\hline
\end{tabular}




\section{Cuadro III \\ Consumo problema de alcohol (RIEsgoso y dañino), por GRUPO de edAd en MUjeres. Instituto Mexicano del Seguro Social, 1994}

\begin{tabular}{|c|c|c|c|c|c|c|c|c|c|c|}
\hline \multirow{4}{*}{$\begin{array}{l}\text { Grupo } \\
\text { de edad }\end{array}$} & \multicolumn{6}{|c|}{ Consumo } & & & & \\
\hline & \multicolumn{2}{|c|}{ Seguro } & \multicolumn{4}{|c|}{ Problema } & & & \multirow{2}{*}{\multicolumn{2}{|c|}{$\begin{array}{c}\text { Encuestas } \\
\text { Total }\end{array}$}} \\
\hline & № & $\%$ & $\frac{P}{1}$ & 0 & No & 0 & $N 0$ & $0 \%$ & & \\
\hline & & & & & & & & & & \\
\hline $12-19$ & 1515 & 95.6 & 59 & 3.7 & 10 & 0.6 & 69 & 4.4 & 1584 & 7.83 \\
\hline $20-24$ & 3386 & 97.3 & 81 & 2.3 & 13 & 0.4 & 94 & 2.7 & 3480 & 17.20 \\
\hline $25-29$ & 3456 & 96.7 & 94 & 2.6 & 23 & 0.6 & 117 & 3.3 & 3573 & 17.66 \\
\hline 30-34 & 3225 & 96.4 & 99 & 3.0 & 21 & 0.6 & 120 & 3.6 & 3345 & 16.53 \\
\hline $35-39$ & 2583 & 96.6 & 78 & 2.9 & 14 & 0.5 & 92 & 3.4 & 2675 & 13.22 \\
\hline 40-44 & 1823 & 96.6 & 43 & 2.3 & 21 & 1.1 & 64 & 3.4 & 1887 & 9.33 \\
\hline $45-49$ & 1200 & 95.6 & 43 & 3.4 & 12 & 1.0 & 55 & 4.4 & 1255 & 6.20 \\
\hline 50-54 & 892 & 96.4 & 24 & 2.6 & 9 & 1.0 & 33 & 3.6 & 925 & 4.57 \\
\hline $55-59$ & 547 & 97.3 & 9 & 1.6 & 6 & 1.1 & 15 & 2.7 & 562 & 2.78 \\
\hline $60-64$ & 443 & 98.0 & 7 & 1.5 & 2 & 0.4 & 9 & 2.0 & 452 & 2.23 \\
\hline $65-69$ & 281 & 96.6 & 8 & 2.7 & 2 & 0.7 & 10 & 3.4 & 291 & 1.44 \\
\hline $70-79$ & 199 & 98.8 & 2 & 1.0 & 2 & 1.10 & 4 & 2.0 & 203 & 1.00 \\
\hline Total & 19550 & 96.6 & 547 & 2.7 & 135 & 0.7 & 682 & 3.4 & 20232 & 100.0 \\
\hline
\end{tabular}

consumo problema de alcohol. La escala de medición AUDIT permite identificar a los sujetos con un consumo de este tipo. Como toda prueba tamiz, no cuenta con una sensibilidad y especificidad de $100 \%$, lo cual implica dejar fuera a individuos con problema en su consumo de alcohol o clasificar erróneamente como tales a sujetos sin un consumo así. ${ }^{10,12,15}$ El uso del AUDIT permite hacer comparativos los diferentes estudios de tamizaje poblacionales dirigidos a identificar sujetos con un consumo problema de alcohol (sea éste un consumo riesgoso o dañino). El AUDIT es el único instrumento que está diseñado específicamente para medirlo. ${ }^{16}$

En México se ha usado el AUDIT en diversos estudios, aunque limitados a grupos de población o regiones geográficas específicos. ${ }^{17}$ Los resultados de este estudio son la primera notificación formal de un estudio nacional poblacional en México, en el cual se empleó el AUDIT como prueba tamiz para el consumo de alcohol. Resulta interesante observar que la prevalencia de consumo riesgoso o dañino que se encontró en el presente trabajo, es similar a la observada por otros autores en la Ciudad de México, en un estudio multinacional que dio origen al empleo del AUDIT en todo el mundo. ${ }^{18}$ En dicho estudio, en pacientes de una unidad de primer nivel de atención, la prevalencia de consumo riesgoso osciló entre 16 y 20\% (similar a 17.7\% observado en el presente estudio) y entre 3 y $6 \%$ en mujeres (similar al observado de $4.5 \%$ ). A pesar de que el instrumento de medición fue distinto, la magnitud observada fue similar.

El AUDIT se ha identificado como una prueba válida en población de ascendencia mexicana que habita en EUA (méxico-americanos), para identificar consumo problema de alcohol. ${ }^{19,20}$ La prevalencia observada en este estudio de consumo riesgoso y dañino de alcohol es mayor que la notificada en población méxico-americana, la cual comparte muchas de las costumbres y estilos de vida con la población mexicana. Mientras que el consumo riesgoso en el presente informe es de $17.7 \%$ y $2.7 \%$ en hombres y mujeres, respectivamente, en los méxico-americanos las prevalencias observadas fueron de $9 \%$ y $2 \%$, en ambos grupos. ${ }^{15}$ A su vez, el consumo dañino en hombres y mujeres del IMSS fue de $4.5 \%$ y $0.7 \%$, mientras que en los méxico-americanos fue menor a $1 \% .{ }^{19}$ Indudablemente esta mayor magnitud del consumo problema de alcohol en la población mexicana resalta el problema de salud, y la necesidad de implantar medidas específicas para su reducción. Hay que considerar que el AUDIT ha mostrado ser un instrumento suficientemente válido (sensible y específico). ${ }^{21,22}$ De hecho, en las mujeres es uno de los instrumentos tamiz con mejor desempeño. ${ }^{23}$

La identificación del consumo problema de alcohol en poblaciones específicas debiera condicionar po- 
líticas de salud orientadas a resolverlo. La clasificación de consumo problema de alcohol, sea riesgoso o dañino, tiene un alto valor predictivo de problemas de salud asociados. ${ }^{10}$ Se ha observado en población mexicana que los ingresos en los servicios de urgencias por accidentes o violencia están fuertemente asociados con el consumo de alcohol, y que el AUDIT puede ser un buen instrumento tamiz en estos sujetos. ${ }^{24}$ De hecho en EUA, la principal causa de muerte en niños y adolescentes son los accidentes de tráfico, una cuarta parte de ellos están relacionados con el consumo de alcohol. Dos terceras partes de estas defunciones involucran a un niño en un vehículo conducido por un sujeto con problemas de alcoholismo, habitualmente el padre o tutor de estos. ${ }^{25}$ Una tercera parte de defunciones en niños son causadas por menores de 21 años (edad legal en ese país para consumir bebidas alcohólicas), que conducían bajo los efectos del alcohol. ${ }^{26}$

La prevalencia de consumo riesgoso en los jóvenes de este estudio es elevada, pues uno de cada cinco a seis hombres menores de 25 años tienen este consumo. Estudios previos ya han identificado al alcoholismo como un importante problema de salud en la población mexicana joven.$^{27}$ Resulta en consecuencia necesario desarrollar políticas y programas de salud específicos, dirigidos a reducir el consumo de alcohol en este grupo poblacional, ya que los hombres jóvenes tienden a estar más frecuentemente involucrados en accidentes fatales de vehículos de motor. ${ }^{26}$ En la etapa productiva es donde el consumo de alcohol alcanza su mayor expresión en esta población. Nuevamente en este grupo de edad estarían indicadas medidas de intervención orientadas a la reducción del consumo riesgoso y dañino de alcohol. La relación del consumo de alcohol con la mortalidad general está bien identificada y el riesgo de morir se incrementa considerablemente en relación directa con el consumo de alcohol. ${ }^{28}$ Se ha identificado que en población adulta, el riesgo de fallecer por enfermedad cerebrovascular se incrementa al doble cuando hay un consumo mayor a 35 copas a la semana. ${ }^{28}$ En la población mexicana se ha observado una asociación de la mortalidad por cirrosis hepática relacionada con el consumo de pulque, bebida que tradicionalmente se ha considerado como de bajo contenido en alcohol. ${ }^{29}$

En la población estudiada de la tercera edad (mayores de 65 años), se observó un descenso en el consumo problema de alcohol. Aun así, uno de cada 10 a 13 hombres de la tercera edad tienen un consumo riesgoso de alcohol. Se ha observado que en este grupo de edad, más de la tercera parte de los ingresos a un hospital se deben a un problema de salud relacionado con el alcohol. ${ }^{30}$
El consumo de alcohol en el país es sin duda alguna heterogéneo. Mientras que en los estados de Chiapas, Colima y Quintana Roo la prevalencia de consumidores problema de alcohol es de 7.5, 8.8 y $9.3 \%$ respectivamente, y en la delegación de Baja California Sur dicha prevalencia alcanza a $28 \%$. En esta última delegación, uno de cada cuatro individuos tiene un consumo problema de alcohol. Aun así la mediana de este consumo se ubica en 13\% de la población general mayor de 12 años, lo cual indudablemente resalta la importancia de este problema de salud poblacional, pobremente identificado en nuestro medio, salvo por sus frecuentes complicaciones médicas.

Se ha predicado el efecto benéfico del consumo moderado de alcohol, tanto para reducir la mortalidad ${ }^{14}$ como la ocurrencia de cardiopatía isquémica, ${ }^{31}$ aunque algunos estudios no han podido documentar este descenso. ${ }^{28}$ En este sentido, las políticas de salud no pueden estar dirigidas a suspender el consumo de alcohol en la población. Parece haber en la actualidad un consenso del efecto benéfico de un consumo moderado. El reto para los sistemas de salud estriba en identificar el límite entre el consumo favorable y el consumo problema de alcohol. La abstinencia sin duda alguna evita todo problema de salud relacionado con el alcoholismo. La identificación de grupos poblacionales con un consumo problema de alcohol debe ser una estrategia permanente de los servicios de salud y el AUDIT puede ser una adecuada prueba tamiz para ello.

\section{Agradecimientos}

Los autores agradecen a los doctores Celia Escandón Romero, Juan Manuel Hernández Ramos, José Daniel Ramírez Galindo y Magdalena Labrandero Iñigo, su colaboración en el diseño del estudio; y a los 36 Coordinadores Delegacionales de Salud Pública del IMSS, su participación en la recolección de la información. La responsabilidad de los resultados que aquí se presentan es exclusivamente de los autores.

\section{Referencias}

1. Lieber CS. Medical disorders of alcoholism. N Engl J Med 1995;33: 1058-1065.

2. Romelsjö A, Karlsson G, Henningso hn L, Jakobsson SW. The prevalence of alcohol-related mortality in both sexes:Variation between indicators, Stockolm, 1987. Am J Public Health 1993;83:838-844.

3. Harford TC. Family history of alcoholism in the United States: Prevalence and demographic characteristics. Br J Addiction 1992;87:931-935. 
4. Grant BF.Alcohol consumption, alcohol abuse and alcohol dependence. The United States as an example.Addiction 1994;89:1357-1365.

5. U mbricht-Schneiter A, Santora P, Moore RD. Alcohol abuse: Comparison of two methods for assessing its prevalence and associated morbidity in hospitalized patients. Am J Med 1991;91:110-118.

6. Yates DW, Hadfield JM, Peters K. The detection of problem drinkers in the accident \& emergency department. Br J Addiction 1987;82:163-167. 7. Secretaría de Salud. Encuesta N acional de Adicciones. México 1998. México, D.F: SSA, 1999:12-18.

8. Secretaría de Salud. Encuesta Nacional de Adicciones. México 1993. México, D.F: SSA, 1994:34,38

9. Tapia R, Medina-Mora ME, Sepúlveda J, De la Fuente JR, kumate J. La Encuesta Nacional de Adicciones de México. Salud Publica Mex 1990; 32:507-522.

10. Conigrave KM, Saunders JB, Rezink RB. Predictive capacity of the AUDIT questionnaire for alcohol - related harm. Addiction 1995;90: 1479-1485.

11. Babor TF, D e la Fuente JR, Saunders J, G rant M.AUDIT the alcohol use disorders identification test: Guidelines for use in primary health care. Ginebra: World Health O rganization, Division of Mental Health, 1989:5,12-15.

12. Conigrave KM, H all W D, Saunders JB.TheAUD IT questionnaire:C hoosing a cut-off score.Addiction 1995; 90:1349-1356.

13. Claussen $B, A$ asaland $O G$. The alcohol use disorders identification test (AUDIT) in a routine health examination of long - term unemployed.Addiction 1993:88:363-368.

14. Doll R, Peto R, Hall E, W heatley K, Gray R. Mortality in relation to consumption of alcohol: 13 years' observations on male British doctors. BMJ 1994;309:911-918.

15. Bohn MJ, Babor TF, Kranzler HR.The alcohol use disorders identification test (AUDIT):Validation of a screening instrument for use in medical settings. J Stud Alcohol 1995;56:423-432.

16. Reid MC, Fiellin DA, O 'Connor PG. Hazardous and harmful alcohol consumption in primary care. Arch Intern Med 1999;159:1681-1689.

17. Medina-Mora E, Carreño S, De la Fuente JR. Experience with the alcohol use disorders identification test (AUDIT) in Mexico. Recent Dev Alcohol 1998;14:383-396

18. Saunders JB, A asaland O G, A mundsen A, G rant M. Alcohol consumption and related problems among primary health care patients:W HO Collaborative project on early detection of persons with harmful alcohol consumption-I. Addiction 1993:88:349-362.
19. Volk RJ, Steinbauer JR, Cantor SB, Holzer CH.The alcohol use disorders identification test (AUDIT) as a screen for at-risk drinking in primary care patients of different racial/ethnic backgrounds. Addiction 1997; 92:197-206

20. Steinbauer JR, Cantor SB, Holzer CE 3rd,Volk RJ. Ethic and sex bias in primary care screening tests for alcohol use disorders. Ann Intern Med 1998; 129:353-362.

21. Rehm J, G reenfield TK,W alsh G, X ie X, Robson L, Single E.Assessment methods for alcohol consumption, prevalence of high risk drinking and harm:A sensitivity analysis. Int J Epidemiol 1999;28:219-224.

22. Saunders JB,A asaland O G, Babor TF, D e la Fuente JR. D evelo pment of the alcohol use disorders identification test (AUDIT):W HO collaborative project on early detection of persons with harmful alcohol consumptionII. Addiction 1993:88:791-804.

23. Bradley KA, Boyd-W ickizer J, Powell Sh, Bruman ML.Alcohol screening questionnaires in women. JAMA 1998;280(2):166-171.

24. Borges G, Medina ME, Cherpitel Ch, Casanova L, Mondragón L, Romero M. Consumo de bebidas alcohólicas en pacientes de los servicios de urgencias de la ciudad de Pachuca, Hidalgo. Salud Publica Mex 1999; 41:3-6.

25. Q uinlan $K P$, Brewer RD, Sleet DA, D ellinger AM. Characteristics of child passenger deaths and injuries involving drinking drivers. JAMA 2000;283(17):2249-2252.

26. Margolis LH, Foss RD, Tolbert W G. Alcohol and motor vehicle - related deaths of children as passengers, pedestrians, and bicyclists. JAMA 2000;283(17):2245-2248.

27. Rojas E, Medina-Mora ME, Juárez F, C arreño S,Villatoro J,Berenzon $S$ et al. El consumo de bebidas alcohólicas y variables asociadas entre los estudiantes de México. Salud Mental 1995;3:23-25.

28. Hart CL, Smith GD, Hole DJ, Hawthorne VM. Alcohol consumption and mortality from all causes, coronary heart disease, and stroke: From a prospective cohort study of Scottish men with 21 years of follow up. BMJ 1999:318:1725-1729.

29. N arro-Robles J, Gutiérrez-Avila H, López-Cervantes M, Borges G, Rosovsky H. La mortalidad por cirrosis hepática en México II. Exceso de mortalidad y consumo de pulque. Salud Publica Mex 1992;34:388-405.

30. A dams W L,Yuan Z, Barboriak JJ, Rimm AA. Alcohol-related hospitalizations of elderly people. JAMA 1993;270:1222-1225.

31. Rimm EB, Klatsky A, Grobbee S, Stampfer MJ. Review of moderate alcohol consumption and reduced risk of coronary heart disease: Is the effect due to beer, wine, or spirits? BMJ 1996:312:731-736. 


\section{Preguntas y alternativas de Respuesta del cuestionario AUDit}

\begin{tabular}{|c|c|c|c|c|c|c|}
\hline & & 0 & 1 & 2 & 3 & 4 \\
\hline 1. & ¿Con qué frecuencia toma bebidas alcohólicas? & N unca & $\begin{array}{l}\text { Cada mes } 0 \\
\text { menos }\end{array}$ & $\begin{array}{l}2 \text { a } 4 \text { veces al } \\
\text { mes }\end{array}$ & $\begin{array}{l}20 \text { más veces } \\
\text { a la semana }\end{array}$ & $\begin{array}{l}4 \text { o más veces } \\
\text { a la semana }\end{array}$ \\
\hline 2. & ¿Cuántas copas toma en un día? & 1 a 2 & 3 a 4 & 5 a 6 & 7 a 9 & 100 más \\
\hline 3. & $\begin{array}{l}\text { ¿Con qué frecuencia toma seis o más copas en una sola } \\
\text { ocasión? }\end{array}$ & $\begin{array}{l}\text { N unca o casi } \\
\text { diario? }\end{array}$ & $\begin{array}{l}\text { Menos de una } \\
\text { vez al mes }\end{array}$ & Una vez al mes & $\begin{array}{l}\text { Una vez a la } \\
\text { semana }\end{array}$ & $\begin{array}{l}\text { Diario o casi } \\
\text { diario }\end{array}$ \\
\hline 4. & $\begin{array}{l}\text { ¿Cuántas veces en el último año notó que una vez que } \\
\text { comenzó a tomar ya no podía parar? }\end{array}$ & $\begin{array}{l}\text { N unca o casi } \\
\text { diario? }\end{array}$ & $\begin{array}{l}\text { Menos de una } \\
\text { vez al mes }\end{array}$ & Una vez al mes & $\begin{array}{l}\text { Una vez a la } \\
\text { semana }\end{array}$ & $\begin{array}{l}\text { Diario o casi } \\
\text { diario }\end{array}$ \\
\hline 5. & $\begin{array}{l}\text { ¿Cuántas veces en el último año el tomar bebidas alco- } \\
\text { hólicas interfirió con sus actividades normales? }\end{array}$ & $\begin{array}{l}N \text { unca o casi } \\
\text { diario? }\end{array}$ & $\begin{array}{l}\text { Menos de una } \\
\text { vez al mes }\end{array}$ & Una vez al mes & $\begin{array}{l}\text { Una vez a la } \\
\text { semana }\end{array}$ & $\begin{array}{l}\text { Diario } 0 \text { casi } \\
\text { diario }\end{array}$ \\
\hline 6. & $\begin{array}{l}\text { ¿Cuántas veces en el último año tuvo que tomar un pri- } \\
\text { mer trago por la mañana para poder funcionar después } \\
\text { de haber tomado el día anterior? }\end{array}$ & $\begin{array}{l}\text { N unca o casi } \\
\text { diario? }\end{array}$ & $\begin{array}{l}\text { Menos de una } \\
\text { vez al mes }\end{array}$ & Una vez al mes & $\begin{array}{l}\text { Una vez a la } \\
\text { semana }\end{array}$ & $\begin{array}{l}\text { Diario o casi } \\
\text { diario }\end{array}$ \\
\hline 7. & $\begin{array}{l}\text { ¿Cuántas veces en el último año tuvo remordimiento o } \\
\text { sentimientos de culpa después de tomar bebidas alco- } \\
\text { hólicas? }\end{array}$ & $\begin{array}{l}\text { N unca o casi } \\
\text { diario? }\end{array}$ & $\begin{array}{l}\text { Menos de una } \\
\text { vez al mes }\end{array}$ & Una vez al mes & $\begin{array}{l}\text { Una vez a la } \\
\text { semana }\end{array}$ & $\begin{array}{l}\text { Diario o casi } \\
\text { diario }\end{array}$ \\
\hline 8. & $\begin{array}{l}\text { ¿C uántas veces en el último año no ha podido recordar } \\
\text { lo que pasó la noche anterior debido a que tomó bebidas } \\
\text { alcohólicas? }\end{array}$ & $\begin{array}{l}\text { N unca o casi } \\
\text { diario? }\end{array}$ & $\begin{array}{l}\text { Menos de una } \\
\text { vez al mes }\end{array}$ & Una vez al mes & $\begin{array}{l}\text { Una vez a la } \\
\text { semana }\end{array}$ & $\begin{array}{l}\text { Diario o casi } \\
\text { diario }\end{array}$ \\
\hline 9. & $\begin{array}{l}\text { Debido a que tomó bebidas alcohólicas, ¿usted o alguien } \\
\text { ha resultado lastimado? }\end{array}$ & No & & $\begin{array}{l}\text { Sí, pero no en } \\
\text { el último año }\end{array}$ & & $\begin{array}{l}\text { Sí, durante el } \\
\text { último año }\end{array}$ \\
\hline & $\begin{array}{l}\text { ¿Existe algún amigo, pariente o médico que conozca su } \\
\text { consumo de bebidas alcohólicas o que le haya sugerido } \\
\end{array}$ & No & & $\begin{array}{l}\text { Sí, pero no en } \\
\text { el último año }\end{array}$ & & $\begin{array}{l}\text { Sí, durante el } \\
\text { último año }\end{array}$ \\
\hline
\end{tabular}
suspenderlo? 